\title{
Exatidão cartográfica de três MDE disponíveis para o Estado de Santa Catarina
}

\author{
Cartographic accuracy of three DEMs available for the State of Santa Catarina
}

\author{
Gustavo Eduardo Pereira ${ }^{1 *}$ \& Alexandre ten Caten ${ }^{2}$ \\ 1 Universidade do Estado de Santa Catarina, Lages, SC, Brasil. *Autor para correspondência: gustavopereira5000@gmail.com. \\ ${ }^{2}$ Universidade Federal de Santa Catarina, Curitibanos, SC, Brasil.
}

Submissão: 18/06/2018 / Aceite: 25/02/2019

\begin{abstract}
RESUMO
O presente estudo objetivou apresentar uma avaliação independente do potencial de um modelo digital de elevação (MDE/SC), gerado por aerofotogrametria, para aplicações as quais demandem escalas detalhadas no Estado de Santa Catarina. Foram coletados 20 pontos de controle e comparados com valores altimétricos derivados do MDE/SC, SRTM v.4.1 e TOPODATA conforme especificações da PECPCD. O MDE/SC atendeu aos requisitos da escala 1:10.000 na classe C, enquanto os MDE SRTM v.4.1 e TOPODATA foram enquadrados na escala 1:50.000 classes A e B, respectivamente. A classificação do $\mathrm{MDE} / \mathrm{SC}$ condiz com a informada no relatório do aerolevantamento e, assim, recomenda-se o emprego do $\mathrm{MDE} / \mathrm{SC}$ em estudos e levantamentos os quais demandem escalas detalhadas no Estado de Santa Catarina.
\end{abstract}

PALAVRAS-CHAVE: geoprocessamento, sensoriamento remoto, SRTM, TOPODATA.

\begin{abstract}
This study aims to present an independent evaluation of the potential of a digital elevation model (DEM/SC), generated by aerophotogrammetry, for applications requiring detailed datasets in the state of Santa Catarina. A total of 20 control points were collected and compared with elevation values derived from DEM/SC, SRTM v.4.1 and TOPODATA according to PEC-PCD. The DEM/SC met the requirements for the $1: 10,000$ scale in class C, while the DEM SRTM v.4.1 and TOPODATA fitted with the scale $1: 50,000$ classes $A$ and $B$, respectively. The DEM/SC classification is consistent with that informed in the aerial survey report. Therefore, one recommends using the DEM/SC in studies and surveys that require detailed elevation datasets in the state of Santa Catarina.
\end{abstract}

KEYWORDS: geoprocessing, remote sensing, SRTM, TOPODATA.

Os Modelos Digitais de Elevação (MDE) são amplamente utilizados em sensoriamento remoto, sistemas de informações geográficas e outras áreas (PENG et al. 2015). O MDE é um plano de informação que descreve a altitude, ponto a ponto, de uma determinada área. Os MDE podem ser gerados a partir de curvas de nível, levantamentos topográficos, sensoriamento remoto ótico, aerofotogrametria, Radio Detection And Ranging (RADAR), Light Amplification by Simulated Emission of Radiation (LASER) e veículos aéreos remotamente pilotados (SHAN \& TOTH 2009).

Em uma meta-análise realizada por PENG et al. (2015) verificou-se que no Brasil foram publicados 180 artigos sobre MDE entre 1994 e 2013. Conforme o estudo, a maior parte das publicações consiste em aplicações dos modelos Shuttle Radar Topography Mission (SRTM) e TOPODATA, que consiste no pósprocessamento dos dados SRTM90 por krigagem com resolução reamostrada para 30 metros. Já no Estado de Santa Catarina, JACQUES et al. (2012) utilizaram o MDE SRTM para o lineamento de estruturas rúpteis em diferentes domínios geológicos na região de Lages. Os autores recomendaram a utilização desse modelo para aplicações em escalas até 1:100.000. Em estudo para detectar condições favoráveis a ocorrência de campos hidromórficos nos municípios de São Joaquim e Urubici, SCHAEFER-SANTOS et al. (2013) analisaram variáveis derivadas do SRTM v.4.1 e concluíram que a escala foi um fator limitante, o que não permitiu a identificação de zonas hidromórficas pequenas. Assim verifica-se que a disponibilidade de um MDE de resolução espacial adequada contribui para os estudos em escalas detalhadas, e a obtenção 
de informações mais acuradas sobre variáveis geomorfológicas.

Em 2010 o Estado de Santa Catarina contratou um levantamento por aerofotogrametria, onde um dos produtos gerados foi o MDE para a totalidade de sua área de $97.037 \mathrm{~km}^{2}$ (doravante denominado MDE/SC). A técnica utilizada foi a geometria epipolar em aerofotos, resultando em um MDE de $1 \mathrm{~m}$ de resolução espacial (SDS 2012). Contudo, esta fonte de dados altimétricos, ao custo de $R \$ 12.000 .000$, é cientificamente subutilizada. A luz de nosso conhecimento, e baseado em uma busca nas bases Scopus, WoS, Scielo, Google Scholar, somente dois estudos, até o presente momento, utilizaram os dados do MDE/SC. Em estudo realizado por MOMO et al. (2016), derivou-se do MDE/SC dados de Height Above Nearest Drainage eficientes para delimitar áreas suscetíveis a inundação na região de Blumenau e Brusque. Nesse estudo, os autores ressaltaram a importância de regularizar as imperfeições do MDE/SC através de ferramentas como fill ou breach. Para fins de mapeamento digital de solos TEN CATEN et al. (2016) avaliaram diferentes procedimentos de interpolação para conversão de um MDE para resolução espacial de $15 \mathrm{~m}$ e ressaltam a importância da avaliação da qualidade desta fonte de dados. Esse estudo também avaliou que o MDE/SC possui ruídos na forma de sinks e spikes, assim, esse MDE requer uma fase de prétratamento antes de sua aplicação. Além disso, conforme os autores, o RMSE vertical do MDE/SC é de, aproximadamente, $1,0 \mathrm{~m}$.

No Brasil, existem poucos levantamentos de grandes áreas como uma cobertura estadual detalhada. O Estado de Pernambuco realizou um aerolevantamento e perfilamento a laser do relevo para todo seu território (SDE/PE 2016). Para permitir aplicações em maiores escalas a geração de dados de alta resolução espacial, faz-se necessária a ampliação desta cobertura pelas demais unidades da federação, bem como posterior avaliação da qualidade dos novos MDE gerados aos moldes deste estudo. Nesse sentido, o Brasil adota como referência o Padrão de Exatidão Cartográfica dos Produtos Cartográficos Digitais (PEC-PCD) (EXÉRCITO BRASILEIRO 2011). O PEC-PCD é indicador estatístico da qualidade posicional que determina os critérios para classificação de trabalhos cartográficos quanto à sua exatidão e à distribuição de erros.

A avaliação da qualidade pode ser realizada pela comparação entre pontos cotados no MDE com pontos cotados provenientes de fontes de maior precisão (e.g. GNSS ou marcos geodésicos) (EXÉRCITO BRASILEIRO 2011). Outra forma é a avaliação independente da exatidão cartográfica a qual elucida características mais desejáveis e contribui na definição dos possíveis empregos do MDE/SC (SDS 2012). Dessa forma, o presente estudo teve como objetivo apresentar uma avaliação independente do potencial do MDE/SC em aplicações as quais demandem escalas detalhadas no Estado de Santa Catarina.

A área de estudo está localizada no município de Frei Rogério, coordenadas centrais $50^{\circ} 49^{\prime} 00^{\prime \prime} \mathrm{W}$ e $27^{\circ} 10^{\prime} 40^{\prime \prime} S$, na microrregião de Curitibanos. Na região predominam formas de relevo ondulado a forteondulado e altitude média de $950 \mathrm{~m}$ (IBGE 2015). Foram coletados 20 pontos por meio do receptor geodésico GPS HiPer Lite+ (Topcon Positioning Systems) de dupla frequência seguindo metodologia empregada por CHAGAS et al. (2010). Os pontos de controle foram coletados em locais representativos da variabilidade espacial do relevo existente na área de estudo, e comparados com valores derivados dos MDE/SC (SDS 2012), SRTM v.4.1 (JARVIS et al. 2008) e TOPODATA (VALERIANO \& ROSSETTI 2012) e a avaliação quantitativa desses MDE seguiu a PEC-PCD (EXÉRCITO BRASILEIRO 2011).

Como o MDE/SC foi disponibilizado na resolução espacial $1 \mathrm{~m}$ foi realizada a conversão de tamanho de pixel para $30 \mathrm{~m} \times 30 \mathrm{~m}$, conforme proposto por TEN CATEN et al. (2016). Desta forma, todos os MDE deste estudo foram comparados na resolução espacial de $30 \mathrm{~m}$. Para assegurar um correto posicionamento geográfico as curvas de nível dos MDE foram sobrepostas, através do software QGIS 2.18.23 com a ferramenta Contorno e posteriormente comparadas, visando eliminar erros sistemáticos de posicionamento horizontal dos pixels. Foi utilizada a ferramenta Rank Filter do software SAGA GIS 4.0.0 com a finalidade de corrigir falhas presentes nos MDE e posteriormente os dados foram submetidos às avaliações da qualidade.

Para cada MDE foram calculados o RMSE, desvio padrão dos erros, teste de normalidade de Shapiro-Wilk, análise de tendência (teste t-student ao nível de $95 \%$ de confiança) e precisão (teste QuiQuadrado) para verificação da porcentagem de pontos que atenderam as especificações da PEC-PCD (EXÉRCITO BRASILEIRO 2011). As classes enquadradas no PEC-PCD, representadas pelas letras A, B, C e $D$ são indicadores da qualidade da exatidão posicional do material cartográfico. Na mesma escala um mapa classe A apresenta menor erro associado e, consequentemente, maior potencial para emprego do MDE em estudos que requerem maior precisão dos dados.

A análise de tendência visa identificar discrepâncias entre o valor considerado verdadeiro e o valor observado (NOGUEIRA JUNIOR 2003), conforme a equação [1]:

$\mathrm{EH}=\mathrm{H}_{\mathrm{GPS}}-\mathrm{H}_{\mathrm{MDE}}$ 
onde: $\mathrm{EH}$ corresponde aos erros na altitude; HGPS é o valor altimétrico de referência (ponto de controle) e HMDE é o valor altimétrico medido no MDE. Após esta etapa foi realizado o cálculo do desvio padrão dos erros $\left(S_{E H}\right)$, conforme a equação 2, em que $\mathrm{EH}$ corresponde a média dos erros e $\mathrm{N}$ ao número de pontos utilizados na avaliação.

$$
\mathrm{S}_{\mathrm{EH}}=\sqrt{\frac{\sum_{i=1}^{\mathrm{n}}\left(E H_{\mathrm{i}}-\mathrm{EH}_{\text {médio }}\right)}{N-1}}
$$

Para cada modelo avaliado foram realizados os testes de tendência, com o objetivo de identificar possíveis erros sistemáticos nos valores de altitude, através do teste de hipótese, conforme segue:

$\mathrm{H}_{0}=\mathrm{EH}_{\text {médio }}=0$ (não é tendencioso)

$\mathrm{H}_{1}=\mathrm{EH}_{\text {médio }} \neq 0$ (é tendencioso)

onde: $\mathrm{EH}_{\text {médio }}$ é a média dos erros. No teste de hipótese calculou-se o valor de "th", conforme a equação 4:

$$
\mathrm{th}_{\mathrm{h}}=\frac{E H_{\text {médio }}}{s_{E H}} \times \sqrt{N}
$$

Foi utilizado o teste "t" de Student, a partir de valores tabelados, a fim de verificar se o valor de "th" está no intervalo de aceitação ou rejeição da hipótese nula. O intervalo de confiança do teste "t" é dado pela equação 5 :

$$
\left|t_{h}\right| t_{n-1 \frac{\alpha}{z}}
$$

Assim, se o valor de "th" for menor que o valor "t" tabelado para $\mathrm{n}-1$ graus de liberdade e nível de significância $\alpha$, os dados estão livre de tendências sistemáticas. Esse tipo de erro esta relacionado com fatores que podem influenciar na coleta de dados, como a copa das árvores, edificações ou interferências atmosféricas.

A análise de precisão foi realizada por meio da comparação entre o desvio padrão $\left(S_{E H}\right)$ e o erro padrão (EP) especificado pelo PEC-PCD (EXÉRCITO BRASILEIRO 2011) para cada escala e classe, conforme:

$$
\begin{aligned}
& H_{0}: S^{2} E H=\sigma^{2} H \\
& H_{1}: S^{2} E H>\sigma^{2} H
\end{aligned}
$$

Em que: $\sigma_{H}$ é o erro padrão pré-estabelecido para cada classe e escala e $S_{E H}$ O desvio padrão dos valores do conjunto de erros de cada MDE avaliado. Após foi aplicada a estatística Qui-Quadrado $\left(\chi^{2}\right)$, de acordo com os Padrões da Exatidão de Produtos Cartográficos Digitais (EXÉRCITO BRASILEIRO 2011) onde foi determinado um intervalo de confiança de $90 \%$ para testes de validação de dados, para isto o cálculo do desvio padrão esperado é realizado conforme a equação 7 :

$$
\chi^{2} \mathrm{H}=\frac{s_{E H}^{2}}{\sigma_{H}^{2}} *(\mathrm{n}-1)
$$

Após foi verificado se o valor de $\mathrm{X}^{2} \mathrm{H}$ se encontrava dentro do intervalo de aceitação conforme a expressão abaixo:

$$
\chi^{2} \mathrm{H} \leq \chi_{(n-1 ; \sigma)}^{2}
$$

Nas equações 7 e 8 o símbolo $\chi^{2}$ denota o resultado obtido pelo teste Qui-Quadrado; $\alpha=0,10$ : (nível de significância) e $n-1$ (graus de liberdade). Quando o valor não atende a expressão acima, a hipótese nula é rejeitada e assume-se que o MDE não atende a precisão pré-estabelecida pela norma (EXÉRCITO BRASILEIRO 2011).

A Tabela 1 apresenta o resumo estatístico dos parâmetros avaliados para os diferentes modelos. Quanto ao RMSE dos MDE avaliados, observa-se que o MDE/SC apresentou os menores valores (2,82 $\mathrm{m})$ similar ao obtido por TEN CATEN et al. (2016). Os MDE SRTM e TOPODATA apresentaram maiores desvios com RMSE de $8,65 \mathrm{~m}$ e $12,92 \mathrm{~m}$, respectivamente. Na literatura há uma ampla variação entre os resultados obtidos para os modelos SRTM e TOPODATA. São encontrados valores da análise de precisão para o SRTM desde 2,9 m (BHANG \& SCHWARTZ 2008); 4,95 m (MORAIS 2017); 5,57 m (SOUZA 2015) 5,95 m (MOURA-BUENO et al. 2016); $7 \mathrm{~m}$ (PINHEIRO 2006); 9,4 m (MICELI et al. 2011); 9,6 m (RODRIGUES et al. 2011) até $11 \mathrm{~m}$ (SANTOS et al. 2006). Para o TOPODATA os valores da análise de precisão variam entre 4,45 m (SOUZA 2015); 8,9 m (MORAIS 2017); 9,78 m (MOURA-BUENO et al. 2016) e 10,9 m (MICELI et al. 2011).

Os dados SRTM e TOPODATA são derivados de um produto em comum, porém os processos utilizados para a geração foram diferenciados, o que obrigatoriamente provocou diferenças entre as bases (BRUBACHER et al. 2012). Essa diferença observada pode estar associada às constantes atualizações do 
MDE SRTM v.4.1, enquanto os dados TOPODATA derivam do SRTM-3" disponível para agosto de 2003 (VALERIANO \& ROSSETTI 2012), além dos erros inerentes aos modelos digitais como erros sistemáticos, picos, depressões e problemas nas interpolações e tamanho de pixel (SOUZA 2015). Quando submetidos ao teste de Shapiro-Wilk todos os MDE apresentaram distribuição normal dos dados (Tabela 1). Em relação à análise de tendência, observa-se que somente o MDE/SC não apresentou tendência ao nível de confiança de $95 \%$.

Tabela 1. Resumo estatístico, análise de tendência e de precisão para os modelos avaliados.

Table 1. Summary statistics, trend and precision analysis for the models evaluated.

\begin{tabular}{|c|c|c|c|c|}
\hline \multirow{2}{*}{\multicolumn{2}{|c|}{ Parâmetros }} & \multicolumn{3}{|c|}{ Modelo Digital de Elevação } \\
\hline & & MDE/SC & SRTM & TOPODATA \\
\hline \multicolumn{2}{|c|}{ Número de pontos } & 20 & 20 & 20 \\
\hline \multicolumn{2}{|c|}{$\mathrm{S}_{E H}(\mathrm{~m})$} & 1,67 & 2,86 & 2,92 \\
\hline \multicolumn{2}{|c|}{ RMSE (m) } & 2,82 & 8,65 & 12,92 \\
\hline \multirow{3}{*}{\multicolumn{2}{|c|}{$\begin{array}{l}\text { Distribuição normal* } \\
\text { Erros grosseiros }\end{array}$}} & --- Resumo est & tico ----- & \\
\hline & & Sim & Sim & Sim \\
\hline & & Não & Não & Sim \\
\hline \multicolumn{2}{|c|}{------------- } & ---- Análise de te & Ência ---. & \\
\hline \multicolumn{2}{|c|}{$\mathrm{t}$ amostral $\left(\mathrm{t}_{\mathrm{h}}\right)$} & $-1,46$ & 3,30 & $-9,70$ \\
\hline \multicolumn{2}{|c|}{$\left|t_{h}\right|<t_{(19,0.05)}$} & Sem tendência & Com tendência & Com tendência \\
\hline \multicolumn{5}{|c|}{--- Análise de precisão1 } \\
\hline \multirow{3}{*}{$\begin{array}{c}\chi^{2} \\
1: 10.000\end{array}$} & Classe B & $\begin{array}{l}\mathrm{x} \\
\mathrm{V}\end{array}$ & $\hat{x}$ & $\begin{array}{l}x \\
x\end{array}$ \\
\hline & Classe C & V & $x$ & $x$ \\
\hline & Classe D & V & $X$ & $\mathrm{x}$ \\
\hline \multirow{4}{*}{$\begin{array}{c}\chi^{2} \\
1: 25.000\end{array}$} & Classe A & V & $\mathrm{X}$ & $\mathrm{X}$ \\
\hline & Classe B & V & V & $\mathrm{V}$ \\
\hline & Classe C & V & V & $\mathrm{V}$ \\
\hline & Classe D & V & V & $\mathrm{V}$ \\
\hline \multirow{4}{*}{$\begin{array}{c}\chi^{2} \\
1: 50.000\end{array}$} & Classe A & V & V & V \\
\hline & Classe B & V & V & V \\
\hline & Classe C & V & V & $\mathrm{V}$ \\
\hline & Classe D & V & V & V \\
\hline \multicolumn{2}{|c|}{ Classe enquadrada PEC } & $\begin{array}{l}1: 10.000-C \\
--C l a s s e s ~ d o s \\
\end{array}$ & $\begin{array}{l}\text { los - } 1: 50.000-A \\
\text { 1:5 }\end{array}$ & $1: 50.000-B$ \\
\hline \multirow{2}{*}{\multicolumn{2}{|c|}{$\begin{array}{l}\text { Classe enquadrada } \chi^{2} \\
\text { Classificação final }\end{array}$}} & $1: 10.000-B$ & $1: 25.000-B$ & $1: 25.000-B$ \\
\hline & & $1: 10.000-C$ & $1: 50.000-A^{* *}$ & $1: 50.000-B^{* *}$ \\
\hline
\end{tabular}

* Teste de normalidade (Shapiro-Wilk), ${ }^{* *} \mathrm{MDE}$ reprovado na análise de tendência, ${ }^{1} \mathrm{~V}=$ Atende aos critérios da classe; ${ }^{1} \mathrm{X}=$ Não atende aos critérios da classe, $\mathrm{S}_{E H}=$ Desvio padrão dos erros, RMSE = Raiz quadrada do erro médio.

$\mathrm{Na}$ análise de precisão (Tabela 1) os MDE SRTM e TOPODATA foram enquadrados na escala 1:50.000, sendo que o SRTM atendeu aos requisitos da classe A e o TOPODATA atendeu à classe B. Nos estudos realizados por MICELI et al. (2011) e SOUZA (2015) os dados do TOPODATA apresentaram resultados similares, enquadrados na Classe $B$ na escala 1:50.000. O MDE SRTM v.4.1 apresentou qualidade altimétrica superior ao TOPODATA nas condições avaliadas, entretanto ambos apresentaram tendência, e no caso do TOPODATA também foram detectados erros grosseiros. O MDE/SC apresentou os melhores resultados, atendendo aos requisitos da escala 1:10.000 da classe $C$, sem erros grosseiros, sem tendência e com distribuição normal dos dados.

A utilização de um MDE de alta resolução com menores erros associados proporciona uma delimitação mais precisa das áreas suscetíveis a inundação no estado de Santa Catarina (MOMO et al. 2016) além de permitir a localização de pequenas áreas hidromórficas de alta relevância ambiental no Parque Nacional de São Joaquim (SCHAEFER-SANTOS et al. 2013).

Assim, verifica-se as potencialidades do MDE/SC, visto que apresenta uma cobertura total do Estado de Santa Catarina, bem como da importância de uma análise independente para destacar possíveis limitações de suas aplicações. Recomenda-se a utilização do MDE/SC para derivar atributos primários e secundários empregados em mapeamentos e levantamentos os quais demandem escalas detalhadas no Estado de Santa Catarina. 


\section{REFERÊNCIAS}

BHANG KJ \& SCHWARTZ F 2008. Limitations in hydrologic applications of C-Band SRTM DEM in low-relief settings. IEEE Geoscience and Remote Sensing Letters 5: 497-501.

BRUBACHER JP et al. 2012. Avaliação de bases SRTM para extração de variáveis morfométricas e de drenagem. Geociências 31: 381-393.

CHAGAS CS et al. 2010. Avaliação de modelos digitais de elevação para aplicação de um mapeamento digital de solos. Revista Brasileira de Engenharia Agrícola e Ambiental 14: 218-226.

EXÉRCITO BRASILEIRO. 2011. Especificação Técnica para a Aquisição de Dados Geoespaciais Vetoriais (ET-ADGV) Versão 2.1.3. Diretoria de Serviço Geográfico do Exército Brasileiro. 254p.

IBGE. 2015. Instituto Brasileiro de Geografia e Estatística. Monografias municipais: Curitibanos, Santa Catarina. Disponível em: <ftp://ftp.ibge.gov.br/Temporario_Edigrafica/monografias_curitibanos.pdf>. Acesso em 15 set. 2018.

JACQUES PD et al. 2012. A comparison for a multiscale study of structural lineaments in southern Brazil: LANDSAT-7 ETM+ and shaded relief images from SRTM3-DEM. Anais da Academia Brasileira de Ciências 84: 931-942.

JARVIS A et al. 2008. Hole-filled SRTM for the globe Version 4, available from the CGIAR-CSI SRTM $90 \mathrm{~m}$ Database. Disponível em: <http://srtm.csi.cgiar.org>. Acesso: em 28 fev. 2018.

MICELI BS et al. 2011. Avaliação vertical de modelos digitais de elevação (MDEs) em diferentes configurações topográficas para médias e pequenas escalas. Revista Brasileira de Cartografia 63: 191-201.

MOMO MR et al. 2016. Desempenho do modelo HAND no mapeamento de áreas suscetíveis à inundação usando dados de alta resolução espacial. Revista Brasileira de Recursos Hídricos 21: 200-208.

MORAIS JD. 2017. Avaliação de modelos digitais de elevação provenientes de dados de sensoriamento remoto de distribuição gratuita. Dissertação (Mestrado em Análise e Modelagem de Sistemas Ambientais). Belo Horizonte: UFMG. 94p.

MOURA-BUENO JM et al. 2016. Assessment of Digital Elevation Model for Digital Soil Mapping in a Watershed with Gently Undulating Topography. Revista Brasileira de Ciência do Solo 40: 1-15.

NOGUEIRA JUNIOR JB. 2003. Controle de qualidade de produtos cartográficos: uma proposta metodológica. Dissertação (Mestrado em Ciências Cartográficas). Presidente Prudente: UNESP. 143p.

PENG Y et al. 2015. Global trends in DEM-related research from 1994 to 2013: a bibliometric analysis. Scientometrics 105: 347-366.

PINHEIRO ES. 2006. Comparação entre dados altimétricos Shuttle Radar Topography Mission, Cartas Topográficas e GPS: numa área com relevo escarpado. Revista Brasileira de Cartografia 58: 1-9.

RODRIGUES TG et al. 2011. Evaluation of the altimetry from SRTM-3 and planimetry from high-resolution PALSAR FBD data for semi-detailed topographic mapping in the Amazon Region. Anais da Academia Brasileira de Ciências 83: 953966.

SANTOS PRA et al. 2006. Avaliação da precisão vertical dos modelos SRTM para a Amazônia. Revista Brasileira de Cartografia 58: 101-107.

SCHAEFER-SANTOS $\mathrm{J}$ et al. 2013. Mapeamento de campos hidromórficos na serra Catarinense por meio de modelagem hidrológica. Floresta e Ambiente 20: 316-326.

SDE/PE. 2016. Secretaria de Desenvolvimento Econômico de Pernambuco. Pernambuco tridimensional: Manual para obtenção dos dados. Disponível em: <http://www.pe3d.pe.gov.br/documentos/manual.pdf>. Acesso em: 16 de set. 2018.

SDS. 2012. Secretaria de Desenvolvimento Econômico e Sustentável. Relatório de produção final: Edital de concorrência pública n0010/2009. Engemap. 202p.

SHAN J \& TOTH C. 2009. Topographic Laser Ranging and Scanning: Principles and Processing. CRC Press. 590p.

SOUZA JOP. 2015. Análise da precisão altimétrica dos modelos digitais de elevação para área semiárida do nordeste brasileiro. Revista do Departamento de Geografia 30: 56-64.

TEN CATEN A et al. 2016. Quality of a digital terrain model for Santa Catarina State. Engenharia Agrícola 36: 12611271.

VALERIANO MM \& ROSSETTI DF. 2012. Topodata: Brazilian full coverage refinement of SRTM data. Applied Geography 32: 300-309. 